\title{
Complementarity of island cross-sea transport links: Bridges, ferries, and mobility in Zhoushan Archipelago, China
}

\section{Adam Grydehøj}

Island and Coastal Zone Institute, Urban and Rural Innovation

Design Research Center, Zhejiang University, China

Island Dynamics, Denmark

agrydehoj@islanddynamics.org

\author{
Huan Zhang \\ School of Civil Engineering and Architecture, Zhejiang \\ University, China \\ 0014979@zju.edu.cn
}

\author{
Publication Information: \\ Received 16 July 2020, Accepted 12 October 2020, Available online 22 December 2020 \\ DOI: $10.21463 /$ jmic.2020.09.2.04
}

\section{Abstract}

Islands and archipelagos are exceptionally dependent on the nature of their transport infrastructure, with cross-sea transport links being of fundamental importance for mobility. Traditionally, the island geography research literature has engaged in a binary and oppositional understanding of the relationship between fixed links such as bridges and tunnels on the one hand and waterborne transport such as ferries on the other. The present paper uses the case of Zhoushan Archipelago (Zhejiang Province, China) to challenge this perception of fixed links and waterborne transport as inherently conflictual by showing how these distinct modes of cross-sea transport have complemented one another and fundamentally altered archipelagic mobilities. We show that even transformative transport infrastructures do not necessarily simply replace existing infrastructures but may instead add to the complexity of the local transport network. In Zhoushan Archipelago, a vast network of new and future inter-island and island-mainland road and rail bridges and tunnels are altering local industry and society as well as the relationship between the archipelago and the mainland, yet ferries remain important for transport between islands and between certain islands and the mainland. We argue that it is fruitful to consider the potential complementarity of different kinds of cross-sea transport links.

\section{Keywords}

archipelagos, bridges, China, ferries, islands, transport links, Zhoushan 


\section{Introduction}

Within transport geography, transport infrastructure is traditionally understood in terms of the manner in which it encourages or discourages, renders more or less accessible or desirable, increases or decreases the capacity of different modes of mobility, or has certain more or less marginal effects on particular economies (e.g. Matas et al., 2015; Gray et al., 2006; Knaap and Oosterhaven, 2003). That is, the geographical impacts of transport infrastructure are largely incremental. They are differences of degree rather than differences of kind. The opening of a new high-speed rail line or automobile expressway between two cities may have major social and economic impacts without fundamentally altering the ways in which places engage with one another. This is the case even for 'rural' communities, which are frequently said to possess special transport geographic characteristics relative to urban communities (e.g. Seedhouse et al., 2016; Gray et al., 2008; Moseley, 1979).

This is not, however, the manner in which transport is typically understood for certain 'isolated' geographical categories, such as mountains, islands, and archipelagos, for which transport infrastructure is often regarded as potentially having fundamental rather than just incremental impacts with regard to mobility. In these cases, the absence or presence of particular transport infrastructures (e.g. roads, bridges, harbours, boats) or restriction of access to these infrastructures for certain social groups (e.g. women, the poor, locals, people living on the other side of the mountain) may indeed have an absolute impact on the ability of individuals to move beyond a certain point, to forge connections across space (Lama, 2018; Ratter, 2018; Mendas, 2015).

There has emerged in the island geography literature a binary and oppositional understanding of the relationship between fixed links (bridges, tunnels, and causeways) and waterborne transport (ferries and private boats) in islands and archipelagos. In the present paper, we use the case of Zhoushan Archipelago (Zhejiang Province, China) (Figure 1) to challenge the perception of fixed links and waterborne transport as inherently conflictual by showing how these distinct modes of cross-sea transport have complemented one another and fundamentally altered archipelagic mobilities as a result. We show that even transformative transport infrastructures do not necessarily simply replace existing infrastructures but may also or instead add to the complexity of local transport networks. Zhoushan Archipelago is not unique in this regard, but the rapid pace of the changes that both the archipelago and its adjacent mainland have undergone over the past two decades makes it powerfully illustrative of the processes under investigation.

Challenging the binary understanding of island and archipelago transport infrastructures is important because the scholarly tendency to position fixed links and waterborne transport as opposing transport strategies and philosophies risks limiting potential visions for how island and archipelago society can or should change over time.

We begin by reviewing and problematising the ways in which the island studies literature has tended to present fixed links and waterborne transport in oppositional terms. We then set forth our methodology for comprehensively mapping and describing Zhoushan Archipelago's cross-sea transport links. Next, we introduce Zhoushan Archipelago and place it in the context of the emerging Yangtze River Delta urban agglomeration, before mapping and describing the archipelago's cross-sea transport links. We then briefly discuss what the composition of Zhoushan Archipelago's transport network can tell us about the interaction between fixed links and waterborne transport. Finally, we present our conclusion and ideas concerning future research. 


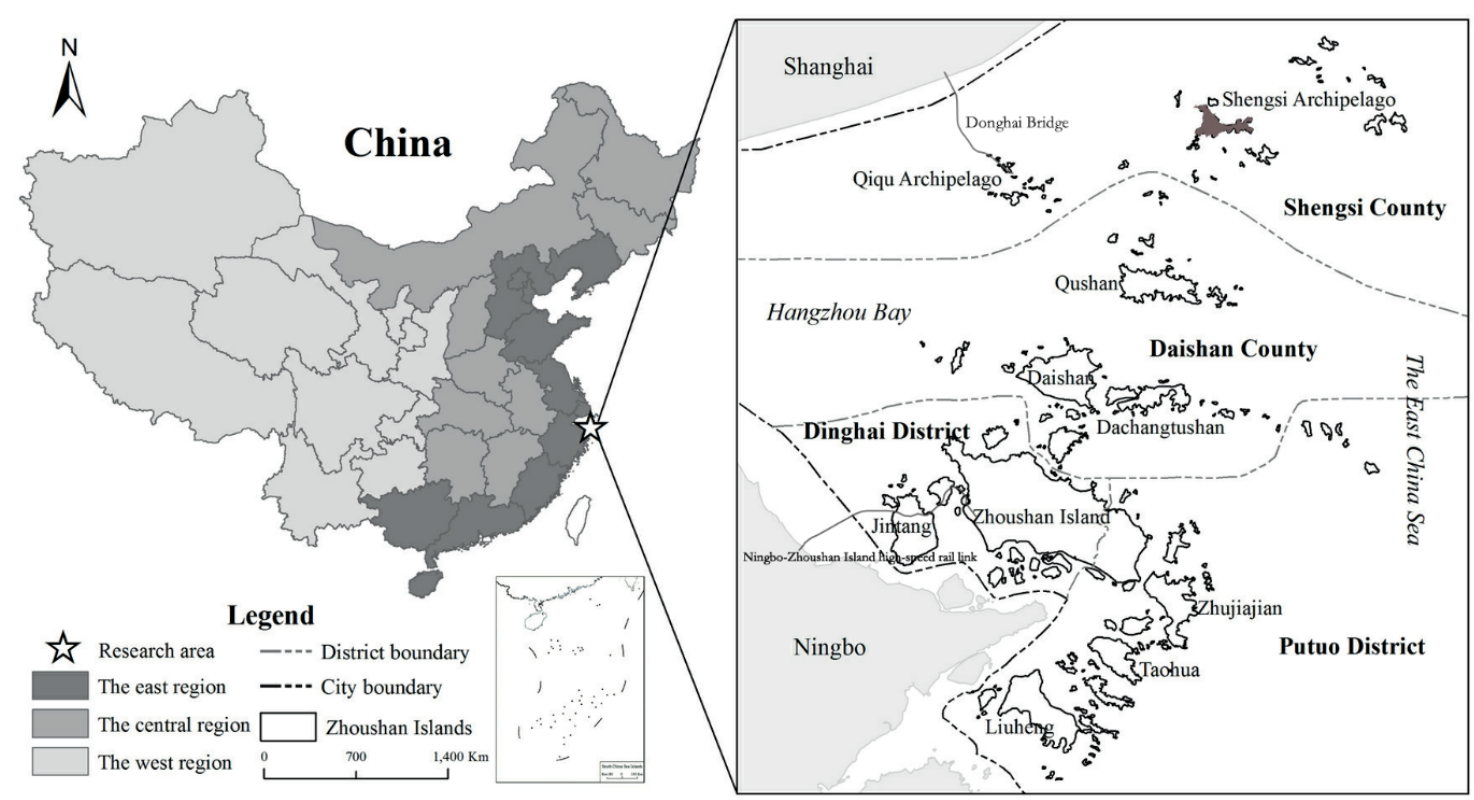

Fig 1. Zhoushan Archipelago's location in China (left) and the archipelago in detail, including bridge connections and jurisdictional divisions (right). (c) Huan Zhang and Shuangshuang Qiu.

\section{Binaries of island transport}

The geographical category of 'island' does not inherently entail remoteness or peripherality. Nevertheless, as has been noted within the field of island studies (Chandler and Pugh, 2020; Hong, 2020a, 2020b, 2017; Krieg, 2018; Pugh, 2018; Grydehøj, 2017; Hayward, 2016), associations of islands with remoteness and peripherality remain common within geographical scholarship, and there is a need for enhanced theoretical consideration of near-shore islands, river islands, islands connected to the mainland by fixed links, and islands as part of anthropogenic processes.

Although literature within island geography and the wider field of island studies has contributed to our understanding of island mobilities, it has tended to reinforce a binary understanding of island modes of transport. That is, even the most nuanced and self-reflective of island studies literature tends to present fixed links and waterborne transport as opposed to one another. Baldacchino $(2020,2007)$ sees the introduction of fixed links to islands as indicative of a mainland policy and economic orientation. Swaminathan (2014) identifies the encroachment of continental connective logics that transform engagement with the sea into a luxury product. Vannini (2011) grasps how, for many islanders, reliance on waterborne transport takes on symbolic importance in explicit opposition to fixed links. Raadik Cottrell and Cottrell (2019) observe a tension between the desire preserve a sense of 'islandness' on the one hand and to make this islandness more accessible through bridging on the other. Grydehøj and Casagrande (2020) regard fixed links as potentially contributing to a particular kind of urban island densification while reliance on waterborne transport can sometimes hold the worst impacts of mass tourism at arm's length. Hayward (2015) sees hard infrastructure as causing island societies to turn inward. Bråthen (2001) questions the ability of fixed links to drive cumulative economic growth in peripheral island communities relative to ferries as a default option for islands. Andersen et al. (2015) find that the replacement of ferries by fixed links may prompt land use change due to increased accessibility but also that it is necessary to consider the characteristics of the place to which an island is being linked. Xie et al. (2018) also find that 
bridging can have major land use impacts, but their analysis takes the alternative (waterborne transport) as an unremarked-upon baseline, which is not subject to analysis. Leung et al. (2017) recognise that ferries and fixed links often exist side by side but present the former as placed at risk by the latter. Sheng et al. (2017) and Lee et al. (2017) problematise the straightforward association of waterborne transport with islandness yet nonetheless present fixed links as a facilitator of an alternative islandness.

Binary understandings of fixed links and waterborne transport are not inevitable, especially given that, as we shall see in the case of Zhoushan Archipelago, it is altogether possible for fixed links and waterborne transport to play complementary - rather than competitive - roles in an island transport network. However, because fixed links and waterborne transport are frequently conceived of as being in conflict, their potential to coexist or even complement one another is often overlooked. This reduces researchers' ability to identify both pitfalls and best practices in island and archipelago transport systems.

We believe that the lack of studies concerning the interaction between coexisting fixed links and waterborne transport has been occasioned in part by a lack of attempts to comprehensively map island and archipelago transport networks. Of the aforementioned papers, only Leung et al. (2017) comprehensively map the ferry routes and fixed links in their study area, categorising small islands into various typologies and considering how these typologies relate to various modes of transport. By mapping and describing the fixed links and ferry routes of Zhoushan Archipelago, here with a focus on how various strands of the archipelagic transport network relate to one another, we seek to contribute to a nonoppositional understanding of fixed links and waterborne transport.

\section{Methods}

The basis for our exploration of Zhoushan Archipelago's transport network is a comprehensive account of all fixed links, scheduled ferry routes, and planned rail links within the archipelago as of spring 2019. As discussed below, Zhoushan Archipelago covers a large area and contains a large number of islands. It furthermore possesses a highly complex system of inter-island and island-mainland ferry routes, which are taking on new significance in light of the construction of island-mainland bridges as well as various inter-island bridges with local orientations.

Although internal transport infrastructures within individual islands (networks of roads, walking paths, bus stations, etc.) are clearly important for understanding how the archipelago's inter-island and island-mainland transport function in practice, we have chosen to limit our study to the cross-sea transport links. By the same token, we have chosen to treat all scheduled ferry routes in the same manner, disregarding whether these are high-speed passenger-only routes, slower passenger-only routes, or ferries that also transport motor vehicles (roll-on/roll-off ferries or otherwise). We have likewise excluded the archipelago's air links, which are significant but are not directly relevant to our analysis.

In order to comprehensively map and describe Zhoushan Archipelago's network of scheduled ferry routes, we have consulted the China Ferry Ticket Network 中國船票網 website. In order to comprehensively map and describe Zhoushan Archipelago's fixed links, we have consulted materials provided by Zhoushan City People's Government as well as manually examined satellite images of the entire archipelago in both Google Maps and Baidu Maps. Because elements of this complex transport network are constantly changing, the quantifications and precise characteristics of the various modes of transport considered here cannot be assumed to remain fixed outside of the study period (March-May 2019) in question. Furthermore, the extreme complexity of the inter-island and island-mainland transport system and a lack of 
available data has prevented us from accurately mapping the precise routes taken by the ferries: our map (Figure 2) shows the points connected by these ferry links but does not attempt to show the path that the ferries follow to get from Point A to Point B.

\section{Case description: Zhoushan Archipelago in the Yangtze River Delta urban agglomeration}

Zhoushan Archipelago is located in the Yangtze River Delta $\left(29^{\circ} 32-31^{\circ} 04^{\prime} \mathrm{N}, 121^{\circ} 30-123^{\circ} 25^{\prime} \mathrm{E}\right)$, spanning the mouth of Hangzhou Bay and stretching between the major cities of Shanghai to the north and Ningbo to the south. With around 1390 islands (103 of which are inhabited year round), a total sea area of 22,000 km², and total land area of $1371 \mathrm{~km}^{2}$, Zhoushan Archipelago is the largest archipelago in China. Besides Zhoushan Island ( $\left.503 \mathrm{~km}^{2}\right)$ itself, there are numerous other large islands (Daishan, Qushan, Zhujiajian, Liuheng, and Jintang) (Yue et al., 2017, 48). The various islands and regions within the archipelago possess different environmental, economic, and social characteristics (Zhang and Xiao, 2020). The 2010 census sets Zhoushan Archipelago's permanent resident population at around 1,121,300 people, though the total population is significantly higher (National Bureau of Statistics, 2011). The prefecture-level city of Zhoushan encompasses four administrative subdivisions: Putuo District, Dinghai District, Shengsi County, and Daishan County.

Although Zhoushan Archipelago has traditionally been regarded as remote and peripheral (Bingenheimer, 2016), it now finds itself in the midst of the Yangtze River Delta urban agglomeration, which has a total population of over 150 million people (Lu et al., 2018) and is composed of 26 interconnected cities (Zhang et al., 2018). The archipelago has experienced rapid economic and population growth over the past two decades, with urbanisation and industrialisation occasioning significant environmental impacts (Wu et al., 2020a, 2020b; Xie et al., 2018; Qiu et al., 2017). The economy has transitioned from heavy dependence on the fishing industry (and thus on small- and medium-sized boats) to a more diverse industrial ecosystem, with port services; marine logistics and technology; education; and nature, heritage, and religious tourism all representing productive growth areas. This has occurred within a development-oriented policy framework that has subjected Zhoushan Archipelago to numerous "strategic plans by the state and local governments" (Pan et al., 2016, p. 216), including the designation of the archipelago as a national 'New Area' in 2011, rendering it a special economic zone focused on the marine economy. The archipelago may be seen as a kind of 'interstitial zone', providing specific urban functions for and acting as an interface between different urban systems (Zhang and Grydehøj, 2020).

\section{Cross-sea transport links in Zhoushan Archipelago}

Zhoushan Archipelago hosts a complex network of cross-sea transport links (Figure 2). Perhaps surprisingly, given the massive investment in fixed links over the past two decades, the archipelago's fixed links consist solely of road bridges of various kinds. There are currently no causeways or tunnels in Zhoushan Archipelago. Work is, however, underway to construct rail links between Zhoushan Archipelago and the mainland, a project that will involve both tunnels and bridges. 


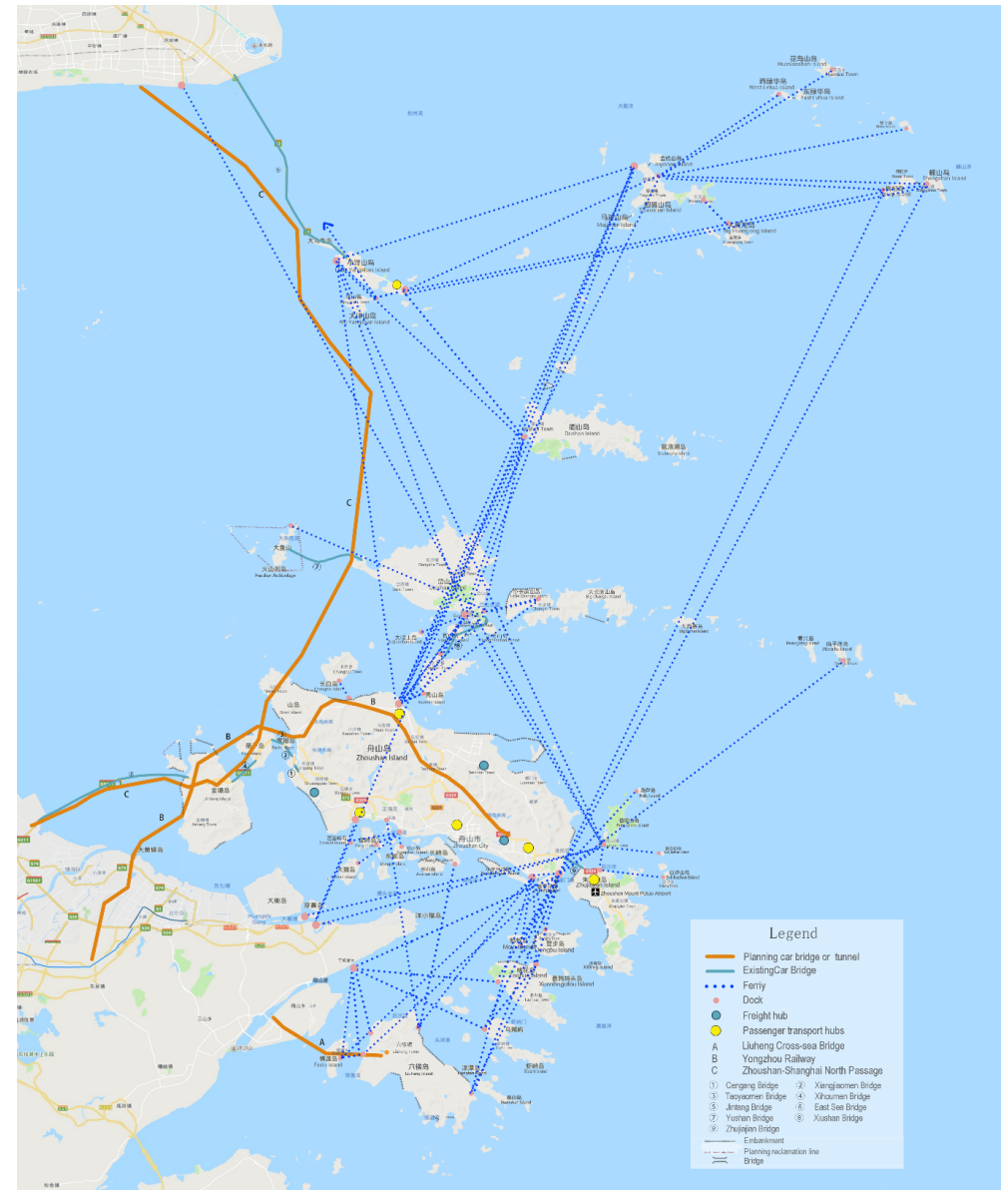

Fig 2. Cross-sea links in Zhoushan Archipelago, March-May 2019. @ Hanning Dong.

\section{Road bridges}

The aforementioned policy framework aimed at promoting Zhoushan Archipelago's development has been constructed alongside significant transport infrastructure development. Zhejiang Province undertook the Zhoushan Island-Mainland Link project, which between 1999 and 2009 involved the construction of a series of five car bridges linking Zhoushan Island with the mainland city of Ningbo (Zhejiang Province). The resultant $68 \mathrm{~km}$ Ningbo-Zhoushan Expressway includes the current third-longest sea bridge in China (Jintang Bridge) as well as the current second-longest suspension bridge in the world (Xihoumen Bridge). The bridge project is not, however, simply intended to facilitate movement between Zhoushan Island and Ningbo, between island and mainland. It also seeks to facilitate movement between mainland cities.

The Zhoushan Island-Mainland Link project is complemented by a parallel and ongoing bridge construction project in the northern portion of the archipelago. Donghai Bridge (32.5 km), connecting Shanghai's Pudong New Area (Shanghai Municipality) with Zhoushan Archipelago's Xiaoyangshan Island, is currently world's second-longest sea bridge (after the newly opened Hong Kong-Zhuhai-Macau Bridge). This bridge will ultimately link up with the bridge network on the islands to the south, significantly shortening transport time between not only Shanghai and Zhoushan Island but also Shanghai 
and Ningbo. Traffic from central Shanghai to the south of Hangzhou Bay and onward to the archipelago must currently travel far out of the way, across Hangzhou Bay Bridge (construction completed 2007).

Bridges have furthermore been constructed to link up various of the smaller islands in Zhoushan Archipelago (see Table 1). There is an increasing tendency for bridges to be built between islands that are connected by bridge to the main Ningbo-Zhoushan Island road network (e.g. the series of bridges linking the islands of Aoshan, Songshan, Changzhi, and Zhoushan), but there are also bridges connecting various smaller islands that do not possess a fixed link to this larger network (e.g. the bridges connecting Xiluhua Island and Dongluhua Island and connecting Sijiaoshan Island and Majishan Island).

Table 1. Cross-sea road bridges in Zhoushan Archipelago, March-May 2019

\begin{tabular}{|c|c|c|c|}
\hline Name & Connecting islands & Length (m) & Construction completed \\
\hline Cengang Bridge & Zhoushan Island-Lidiao Island & 793 & 2001 \\
\hline Xiangjiaomen Bridge & Lidiao Island -Fuchi Island & 951 & 2002 \\
\hline Taoyaomen Bridge & Fuchi Island-Cezi Island & 888 & 2003 \\
\hline Xihoumen Bridge & Cezi Island-Jintang Island & 1650 & 2009 \\
\hline Jintang Bridge & Jintang Island-Ningbo City & 21020 & 2009 \\
\hline Donghai Bridge & Xiaoyangshan Island-Shanghai City & 32500 & 2005 \\
\hline Yushan Bridge & Daishan Island-Yushan Island & 8815 & 2018 \\
\hline Xiushan Bridge & Daishan Island-Xiushan Island & 3063 & 2018 \\
\hline Changzhi Bridge & Changzhi Island-Songshan Island & 580 & 2007 \\
\hline Aoshan Bridge & Songshan Island-Aoshan Island & 343 & 2007 \\
\hline Lujiazhi Bridge & Zhoushan Island-Lujiazhi Island & 1400 & 2006 \\
\hline Qingren Island Bridge & Qingren Island-Zhujiajian Island & 122 & 1997 \\
\hline Jiangnan Bridge & Daishan Island-Jiangnanshan Island & 599 & 2009 \\
\hline Xianzhou Bridge & Daishan Island-Shanwaishan Island & 91.84 & 2004 \\
\hline Jinqing Bridge & Sijiaoshan Island-Jinjishan Island & 600.4 & 2001 \\
\hline Sanjiaojiang Bridge & Shengshan Island-Gouqi Island & 781 & 2010 \\
\hline Luhua Bridge & Dongluhua Island-Xiluhua Island & 172 & 1972 \\
\hline Majishan Bridge & Sijiaoshan Island-Majishan Island & 210 & 1996 \\
\hline Guanshan Bridge & Guanshan & 926 & 2014 \\
\hline
\end{tabular}

\section{Ferries}

Zhoushan Archipelago hosts a network of 143 ferry routes (Tables 2-5). Both the most frequent and least frequent of these routes involve the island of Putuoshan, a major tourism site: ferries depart from Zhoushan Island Banshengdong Dock to Putuoshan up to 33 times per day, and ferries depart from Shanghai Wusong Dock to Putuoshan three times per week. The presence of the Ningbo-Zhoushan Expressway has sharply reduced ferry travel from the bridge-linked islands to the mainland, yet the ferry network otherwise remains fairly comprehensive and robust. A number of harbours function as hubs for connecting small islands from various parts of the archipelago with one another. Thus, for example, 
in order to travel anywhere via public transport, residents of Miaozihu Island (Putuo District) are dependent on four weekly ferry trips (120 minutes) to and from Zhoushan Island Banshengdong Dock.

Zhoushan Archipelago's ferry network was formerly run by a number of different companies, but the ferry services have been consolidated into a single state-owned enterprise, allowing for cross-subsidisation and strong economies of scale.

Table 2. Scheduled ferry routes in Putuo District, Zhoushan Archipelago, March-May 2019

\begin{tabular}{|c|c|c|c|c|c|c|c|c|c|c|c|}
\hline Dep. term. & Arr. term. & $\begin{array}{l}\text { Dep. } \\
\text { /day }\end{array}$ & $\begin{array}{l}\text { Travel } \\
\text { (min.) }\end{array}$ & Dep. term. & Arr. term. & $\begin{array}{l}\text { Dep. } \\
\text { /day }\end{array}$ & $\begin{array}{l}\text { Travel } \\
\text { (min.) }\end{array}$ & Dep. term. & Arr. term. & $\begin{array}{l}\text { Dep. } \\
\text { /day }\end{array}$ & $\begin{array}{l}\text { Travel } \\
\text { (min.) }\end{array}$ \\
\hline \multirow[t]{12}{*}{$\begin{array}{l}\text { Zhoushan } \\
\text { Banshengdong }\end{array}$} & $\begin{array}{l}\text { Taohua } \\
\text { Maocaowu }\end{array}$ & 6 & 60 & \multirow[t]{9}{*}{$\begin{array}{l}\text { Zhoushan } \\
\text { Duntou }\end{array}$} & $\begin{array}{l}\text { Taohua } \\
\text { Maocaowu }\end{array}$ & 6 & $20-40$ & \multirow[t]{3}{*}{$\begin{array}{l}\text { Liuheng } \\
\text { Daao }\end{array}$} & $\begin{array}{l}\text { Zhoushan } \\
\text { Duntou }\end{array}$ & 8 & $40-70$ \\
\hline & Mayi & 4 & 40 & & Mayi & 5 & $20-40$ & & Changzhi & 5 & 60 \\
\hline & Liuheng Taimen & 3 & 70 & & Liuheng Daao & 10 & $40-70$ & & $\begin{array}{l}\text { Liuheng } \\
\text { Zhangqigang }\end{array}$ & 5 & 45 \\
\hline & $\begin{array}{l}\text { Xiazhi } \\
\text { Shanpeng }\end{array}$ & 6 & 60 & & Liuheng Taimen & 5 & $50-80$ & \multirow[t]{2}{*}{$\begin{array}{l}\text { Liuheng } \\
\text { Taimen }\end{array}$} & $\begin{array}{l}\text { Taohua } \\
\text { Maocaowu }\end{array}$ & 2 & 78 \\
\hline & Dengbu & 4 & 30 & & Xiazhi & 5 & $35-75$ & & $\begin{array}{l}\text { Zhoushan } \\
\text { Duntou }\end{array}$ & 3 & 80 \\
\hline & Shengshan & 1 & 240 & & $\begin{array}{l}\text { Xiazhi } \\
\text { Shanpeng }\end{array}$ & 1 & 60 & $\begin{array}{l}\text { Liuheng } \\
\text { Zhangqigang }\end{array}$ & Fodu & 5 & 15 \\
\hline & Hulu & $\begin{array}{l}4 \\
\text { /week }\end{array}$ & 110 & & Dengbu & 4 & 25 & $\begin{array}{l}\text { Liuheng } \\
\text { Shaoao }\end{array}$ & Ningbo Guoju & 17 & 30 \\
\hline & Putuoshan & $8-33$ & $15-25$ & & Fodu & 1 & 150 & \multirow[t]{2}{*}{ Mayi } & $\begin{array}{l}\text { Zhoushan } \\
\text { Banshengdong }\end{array}$ & 4 & 40 \\
\hline & Miaozihu & 4 & 120 & & Gouqi & 1 & 300 & & $\begin{array}{l}\text { Taohua } \\
\text { Maocaowu }\end{array}$ & 6 & 15 \\
\hline & Liuheng Daao & 9 & 70 & \multirow[t]{2}{*}{ Xiazhi } & Ningbo Guoju & 1 & 70 & Dengbu & $\begin{array}{l}\text { Zhoushan } \\
\text { Banshengdong }\end{array}$ & 4 & 30 \\
\hline & Gouqi & 1 & 270 & & $\begin{array}{l}\text { Zhoushan } \\
\text { Banshengdong }\end{array}$ & 8 & $80-120$ & \multirow[t]{3}{*}{$\begin{array}{l}\text { Taohua } \\
\text { Maocaowu }\end{array}$} & Liuheng Taimen & 2 & 78 \\
\hline & Fodu & 1 & 90 & \multirow[t]{2}{*}{ Zhujiajian } & Putuoshan & 80 & $10-15$ & & $\begin{array}{l}\text { Zhoushan } \\
\text { Banshengdong }\end{array}$ & 4 & 20 \\
\hline \multirow[t]{9}{*}{ Putuoshan } & $\begin{array}{l}\text { Shanghai } \\
\text { Nanpu Bridge }\end{array}$ & 1 & 360 & & Baisha & 4 & 30 & & Mayi & 6 & \\
\hline & Ningbo Baifeng & 1 & 70 & Hulu & $\begin{array}{l}\text { Zhoushan } \\
\text { Duntou }\end{array}$ & 1 & 110 & $\begin{array}{l}\text { Taohua } \\
\text { Shaao }\end{array}$ & Ningbo Guoju & 1 & \\
\hline & Ningbo Daxie & $9-12$ & 70 & Fodu & $\begin{array}{l}\text { Liuheng } \\
\text { Zhangqigang }\end{array}$ & 5 & 15 & & & & \\
\hline & $\begin{array}{l}\text { Zhoushan } \\
\text { Banshengdong }\end{array}$ & 38 & $15-25$ & Baisha & Zhujiajian & 4 & 30 & & & & \\
\hline & Zhujiajian & 80 & $10-15$ & Miaozihu & $\begin{array}{l}\text { Zhoushan } \\
\text { Banshengdong }\end{array}$ & 4 & 120 & & & & \\
\hline & $\begin{array}{l}\text { Shanghai } \\
\text { Wusong }\end{array}$ & $\begin{array}{l}3 \\
\text { /week }\end{array}$ & 720 & Luojiashan & Putuoshan & 2 & 30 & & & & \\
\hline & $\begin{array}{l}\text { Daishan } \\
\text { Gaoting }\end{array}$ & 1 & 150 & & & & & & & & \\
\hline & Xiaoyangshan & 6 & 270 & & & & & & & & \\
\hline & Luojiashan & 2 & 30 & & & & & & & & \\
\hline
\end{tabular}


Table 3. Scheduled ferry routes in Dinghai District, Zhoushan Archipelago, March-May 2019.

\begin{tabular}{|c|c|c|c|c|c|c|c|}
\hline Dep. term. & Arr. term. & Dep./day & $\begin{array}{l}\text { Travel } \\
\text { (min.) }\end{array}$ & Dep. term. & Arr. term. & Dep./day & $\begin{array}{l}\text { Travel } \\
\text { (min.) }\end{array}$ \\
\hline $\begin{array}{l}\text { Zhoushan } \\
\text { Yadanshan }\end{array}$ & Ningbo Baifeng & 16 & 45 & \multirow[t]{4}{*}{$\begin{array}{l}\text { Zhoushan Dinghai } \\
\text { Passenger Terminal }\end{array}$} & Damao & 2 & 40 \\
\hline \multirow{7}{*}{$\begin{array}{l}\text { Zhoushan } \\
\text { Sanjiang }\end{array}$} & Xiushan Lanshan & 10 & 20 & & Xixiezhi & 3 & 30 \\
\hline & Sijiaoshan Lizhushan & 4 & $120-150$ & & Panzhi Island & 11 & 30 \\
\hline & Daishan Gaoting & 20 & 60 & & Zhoushan Xiaoju & 3 & 20 \\
\hline & Daishan Gaoting New Town & 3 & 75 & Damao & $\begin{array}{l}\text { Zhoushan Dinghai } \\
\text { Passenger Terminal }\end{array}$ & 2 & 40 \\
\hline & Xiaoyangshan & 1 & 150 & Xixiezhi & $\begin{array}{l}\text { Zhoushan Dinghai } \\
\text { Passenger Terminal }\end{array}$ & 3 & 30 \\
\hline & Changtu & 5 & 60 & Panzhi & $\begin{array}{l}\text { Zhoushan Dinghai } \\
\text { Passenger Terminal }\end{array}$ & 11 & 30 \\
\hline & Qushan & 8 & $60-150$ & Changbai & Zhoushan Haifeng & 10 & 10 \\
\hline $\begin{array}{l}\text { Zhoushan } \\
\text { Xiaoju }\end{array}$ & $\begin{array}{l}\text { Zhoushan Dinghai Passenger } \\
\text { Terminal }\end{array}$ & 2 & 20 & Changzhi & Liuheng Daao & 5 & 10 \\
\hline $\begin{array}{l}\text { Liuheng } \\
\text { Zhangqigang }\end{array}$ & Liuheng Daao & 5 & 45 & Zhoushan Haifeng & Changbai & 10 & 10 \\
\hline
\end{tabular}

Table 4. Scheduled ferry routes in Shengsi County, Zhoushan Archipelago, March-May 2019.

\begin{tabular}{|c|c|c|c|c|c|c|c|c|c|c|}
\hline Dep. term. & Arr. term. & Dep./day & $\begin{array}{l}\text { Travel } \\
\text { (min.) }\end{array}$ & Dep. term. & Arr. term. & Dep./day & $\begin{array}{l}\text { Travel } \\
\text { (min.) }\end{array}$ & Dep. term. & Arr. term. & Dep./day \\
\hline \multirow[t]{8}{*}{$\begin{array}{l}\text { Sijiaoshan } \\
\text { Lizhushan }\end{array}$} & Daishan Gaoting & 1 & 150 & \multirow[t]{3}{*}{ Gouqi } & $\begin{array}{l}\text { Xiaoyangshan } \\
\text { Shenjiawan }\end{array}$ & 2 & 145 & Shengshan & $\begin{array}{l}\text { Zhoushan } \\
\text { Duntou }\end{array}$ & 1 \\
\hline & Qushan & 2 & 70 & & $\begin{array}{l}\text { Sijiaoshan } \\
\text { Caiyuan Town }\end{array}$ & 2 & 60 & & $\begin{array}{l}\text { Sijiaoshan } \\
\text { Caiyuan Town }\end{array}$ & 1.5 \\
\hline & $\begin{array}{l}\text { Daishan Gaoting } \\
\text { New Town }\end{array}$ & 1 & 110 & & $\begin{array}{l}\text { Zhoushan } \\
\text { Banshengdong }\end{array}$ & 1 & 270 & & $\begin{array}{l}\text { Xiaoyangshan } \\
\text { Shenjiawan }\end{array}$ & 1 \\
\hline & $\begin{array}{l}\text { Zhoushan } \\
\text { Sanjiang }\end{array}$ & 2 & 150 & Wulong & Huanglong & 7 & 20 & $\begin{array}{l}\text { Sijiaoshan } \\
\text { Caiyuan } \\
\text { Town }\end{array}$ & Huaniao & 1.5 \\
\hline & Xiaoyangshan & 5 & 80 & Huanglong & Wulong & 7 & 20 & & Shengshan & 1.5 \\
\hline & Dayangshan & 3 & 60 & \multirow{4}{*}{$\begin{array}{l}\text { Xiaoyangshan } \\
\text { Shenjiawan }\end{array}$} & Gouqi & 3 & 145 & & Lvhua & 0.5 \\
\hline & $\begin{array}{l}\text { Shanghai Nanpu } \\
\text { Bridge }\end{array}$ & 8 & $\begin{array}{l}70- \\
150\end{array}$ & & $\begin{array}{l}\text { Shanghai } \\
\text { Nanpu Bridge }\end{array}$ & 2 & 100 & & Bixia & 1 \\
\hline & Ningbo Zhenhai & 3 & 180 & & Dayangshan & 1 & 40 & & Gouqi & 1.5 \\
\hline \multirow[t]{3}{*}{ Dayangshan } & Xiaoyangshan & 6 & 20 & & Shengshan & 1 & 155 & Huaniao & $\begin{array}{l}\text { Sijiaoshan } \\
\text { Caiyuan Town }\end{array}$ & 1.5 \\
\hline & $\begin{array}{l}\text { Xiaoyangshan } \\
\text { (Emergeny) }\end{array}$ & 2 & 30 & \multirow[t]{2}{*}{ Xiaoyangshan } & Qushan & 2 & 70 & Bixia & $\begin{array}{l}\text { Sijiaoshan } \\
\text { Caiyuan Town }\end{array}$ & 1 \\
\hline & $\begin{array}{l}\text { Xiaoyangshan } \\
\text { Shenjiawan }\end{array}$ & 1 & 15 & & $\begin{array}{l}\text { Daishan } \\
\text { Gaoting }\end{array}$ & 2 & 105 & Lvhua & $\begin{array}{l}\text { Sijiaoshan } \\
\text { Caiyuan Town }\end{array}$ & 1 \\
\hline
\end{tabular}


Table 5. Scheduled ferry routes in Daishan County, Zhoushan Archipelago, March-May 2019.

\begin{tabular}{|c|c|c|c|c|c|c|c|}
\hline Dep. term. & Arr. term. & Dep./day & $\begin{array}{l}\text { Travel } \\
\text { (min.) }\end{array}$ & Dep. term. & Arr. term. & Dep./day & $\begin{array}{l}\text { Travel } \\
\text { (min.) }\end{array}$ \\
\hline \multirow{2}{*}{$\begin{array}{l}\text { Xiushan } \\
\text { Lanshan }\end{array}$} & Daishan Gaoting & 6 & 25 & \multirow[t]{4}{*}{ Qushan } & Zhoushan Sanjiang & 8 & $60-150$ \\
\hline & Zhoushan Sanjiang & 10 & 20 & & $\begin{array}{l}\text { Daishan Gaoting New } \\
\text { Town }\end{array}$ & 7 & 70 \\
\hline Xiushan Xiubei & Daishan Gaoting & 6 & 15 & & Sijiaoshan Lizhushan & 1 & 70 \\
\hline \multirow[t]{10}{*}{ Daishan Gaoting } & $\begin{array}{l}\text { Shanghai Nanpu } \\
\text { Bridge }\end{array}$ & 2 & 200 & & Xiaoyangshan & 2 & 70 \\
\hline & Yushan & 1 & $60-80$ & \multirow[t]{2}{*}{ Changtu } & $\begin{array}{l}\text { Daishan Gaoting New } \\
\text { Town }\end{array}$ & 9 & 15 \\
\hline & Qushan & 6 & 45 & & Zhoushan Sanjiang & 5 & 60 \\
\hline & Changtu & 9 & 25 & \multirow{7}{*}{$\begin{array}{l}\text { Daishan Gaoting New } \\
\text { Town }\end{array}$} & Qushan & 7 & 70 \\
\hline & Sijiaoshan Lizhushan & 1 & 150 & & Changtu & 9 & 15 \\
\hline & Zhoushan Sanjiang & 20 & 60 & & Sijiaoshan Lizhushan & 3 & 110 \\
\hline & Xiushan Xiubei & 6 & 15 & & Zhoushan Sanjiang & 3 & 75 \\
\hline & Xiushan Lanshan & 6 & 25 & & & & \\
\hline & Dajiaoshan & 1 & 15 & & & & \\
\hline & Xiaoyangshan & 2 & 105 & & & & \\
\hline
\end{tabular}

\section{Rail links}

Zhoushan Archipelago currently possesses no rail links. However, there are plans for a number of such links in the future. Work has recently begun on a Zhoushan Island-Ningbo high-speed rail link $(77 \mathrm{~km}$, including various bridges and $16.2 \mathrm{~km}$ of tunnel), which will reduce travel time between Zhoushan Island and Ningbo to 30 minutes and travel time between Zhoushan Island and the provincial capital Hangzhou to 75 minutes (China Daily, 2019). This rail link will cater significantly to tourist demand but will also be able to carry freight.

The Zhoushan Islands New District (City) Master Plan (2012-2030) 浙江舟山群岛新区（城市）总体规划 (2012-2030) furthermore sets forth future plans for the construction of an archipelago-traversing rail link between Shanghai and Ningbo (Zhoushan City Housing and Urban-Rural Development Bureau, 2015).

\section{Discussion}

The construction of fixed links such as bridges, causeways, and tunnels that connect islands to the mainland or to larger islands is frequently associated in the island geography literature with the loss of existing ferry services and waterborne transport options (Leung et al., 2017; Baldacchino, 2007; Knowles, 2000).

The experience of Zhoushan Archipelago has been more complex. Although some individual ferry routes have been lost, and others have become less frequent, the introduction of fixed links has not led to the general disappearance of intraarchipelago ferry routes. This is perhaps in part due to the island-mainland transport orientation of the major fixed link projects, which means that ferries continue to serve local needs in a particular manner, connecting the places between 
which islanders need to travel in their daily lives. In addition, it is possible that the increase in resident and visitor numbers to Zhoushan occasioned by the bridges has increased demand for some ferry services, with ferries between Putuoshan and other islands clearly benefiting from the road access between the mainland and Zhoushan Island and Xiaoyangshan Island respectively.

Although the introduction of fixed links has not led to the widespread discontinuation of ferry routes, as Figure 2 shows, there is not a great deal of redundancy between the ferry routes and the existing bridge network. Furthermore, those small islands that lacked ferry links in the past largely continue to lack both ferry links and fixed links: there remain inhabited islands that are inaccessible by public transport. In other words, the fixed links have brought the islands that were already most 'central' to the archipelago closer to the mainland but have only indirectly affected the archipelago's most peripheral islands.

It will be interesting to observe how or whether the provision of waterborne transport will change with the further construction of the road bridges stretching from Shanghai to Xiaoyangshan Island to Daishan Island to Zhoushan Island and eventually on to Ningbo as well as the high-speed rail link between Ningbo and Zhoushan Island. It is conceivable that even as the archipelago becomes increasingly connected to the mainland, its needs for ferries will remain stable or even rise. Once the road bridge project is complete, Zhoushan will be fully integrated into the Yangtze River Delta expressway network, with the bridges serving the needs of island-mainland and mainland-island-mainland passenger and freight transport while the ferries provide opportunities for much of the archipelago's internal transport needs.

More generally, Zhoushan's existing two sets of archipelago-mainland bridges have made possible the rapid urbanisation of and growth of heavy industry in Zhoushan Archipelago. Xiaoyangshan Island is the site of Yangshan Deep-Water Port, the key component of Port of Shanghai, which is far and away the busiest container port in the world by volume, handling 42 million teu in 2018 (Si, 2019b). Meanwhile, Ningbo-Zhoushan Port, which is substantially located on and around Zhoushan Island, is the third-busiest container port in the world by volume, handling 28 million teu in 2018 (Si, 2019a). The growth of the marine economy in Zhoushan Archipelago has led to a substantial rise in the islands' population over the past couple of decades, though this has also coincided with increasing population concentration on Zhoushan Island (Yue et al., 2017). Despite being demographically and economically dwarfed by the numerous major cities in the Yangtze River Delta urban agglomeration, the archipelago has - far from becoming a backwater - been thoroughly integrated into the wider regional and national economy and in fact plays a number of unique roles in this economy (Zhang and Grydehøj, 2020).

It would be an oversimplification to say, however, that ferries are for locals while fixed links are for visitors. As Gillis (2004) notes, ferries are particularly popular with island tourists and other non-residents, for whom physical disconnection from the mainland may serve as a positive marker of difference. As such, the desire of tourists from major cities on the mainland to reach Zhoushan Island quickly and conveniently does not necessarily translate to a desire for all transport within the archipelago to be equally rapid. For example, the island of Putuoshan (Figure 3 ) is eminently bridgeable from Zhoushan Island or Zhujiajian Island, but the disadvantages of creating a fixed link to this major site for religious and cultural heritage tourism would likely outweigh its benefits: Putuoshan's sense of attractive remoteness is boosted by its only being accessible by boat. Thus, while the numbers of tourists to both Zhujiajian and Putuoshan have risen as a result of the bridge from Zhoushan Island to Zhujianjian (Xie et al., 2019), a bridge straight to Putuoshan itself would probably not be worthwhile. 


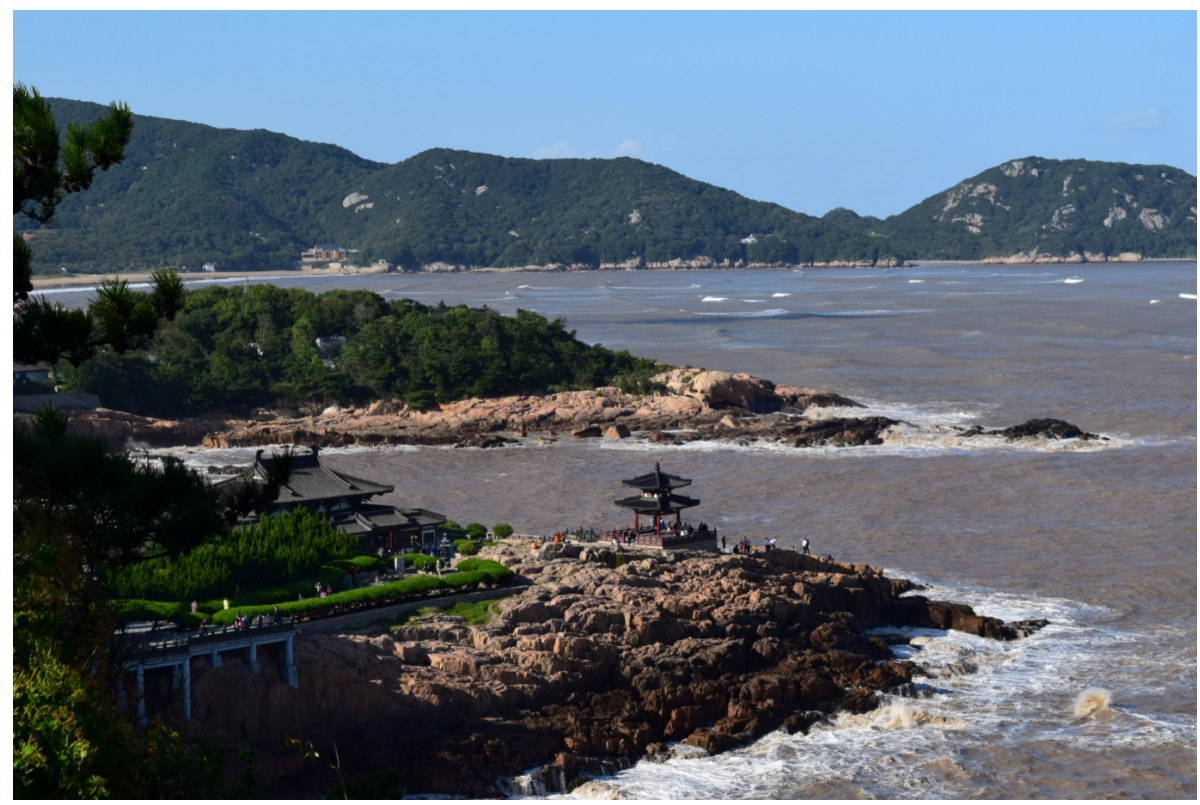

Fig 3. Putuoshan, a major cultural heritage and religious tourism destination, benefits from its lack of bridges. @ Adam Grydehøj, 2018.

By the same token, the integration of Zhoushan Archipelago into the Yangtze River Delta urban agglomeration does not merely allow the penetration of the archipelago by mainland business and industrial actors; it also provides islanders with greater opportunities to lead fulfilling lives without having to make the difficult choice between maintaining strong island links and pursuing educations, careers, and personal relationships elsewhere (see discussion in Cooke and Petersen, 2019).

Our analysis of ferry and bridge interactions in Zhoushan Archipelago reflects, of course, the fact that this archipelago possesses a highly complex transport network. A similar analysis in a different archipelago (fewer islands, less populous, greater population concentration, farther from the continental coast, farther from major mainland population centres, in a state system that provides less support for public transport, etc.) might produce very different results. For example, while Grydehøj and Hayward's (2014) analysis of intra-archipelagic and island-mainland mobility in the Isles of Scilly and the Isle of Wight (England) suggests that lack of transport options may have a protective effect on island economies, the islands in question differ strongly not only from one another but also from Zhoushan Archipelago in terms of topology, industrial composition, population, and practical government capacity to implement policy.

This, however, is precisely the point. Some scholarly preconceptions regarding the relationships between fixed links and ferries may be rooted in the fact that they have arisen from very different geographical contexts (Grydehøj, 2020). If the dominant theories regarding these relationships were developed in the context of islands in specific parts of Europe and North America, the result may be an unintentional provincialism, closing off understanding of other potential combinations of island and archipelago transport modes.

As Castanho et al. (2020, p. 2) note in their discussion of archipelagic transport infrastructure, "insularity characterizes the general 'atmosphere' that determines the experiential identity of the 'islander' and the distinct environment and trends created in these geographic regions." Perceptions of islandness as well as reactions to and impacts of various forms of connective infrastructure are mutually contingent and must be considered in terms of development over time. 
Bridges are often seen as presenting a risk to sense of island place (Raadik Cottrell and Cottrell, 2019). However, factors such as depopulation caused by lack of opportunity and dwindling ability or desire to pursue traditional livelihoods also often present a risk to people living their lives as 'islanders'. Indeed, Zhang and Grydehøj (2020) find that, for some Zhoushan islanders, the archipelago's increasing links with the mainland have not only provided new economic opportunities but also helped emphasise and cause people to value what makes the archipelago unique. This does not mean that bridging islands is always good; it just means that there is no straightforward relationship between island vitality, sense of island place, and archipelago relations on the one hand and form of transport or connective infrastructure on the other. The task of evaluating island sustainability is complex (Chen and Dong, 2020).

\section{Conclusion}

In this study, we have comprehensively mapped and described the cross-sea transport links in Zhoushan Archipelago. We have done so in order to shed light on the interrelationships between fixed links and waterborne transport in an archipelago, adding nuance to the often binary discourse concerning island and archipelago transport networks. Rather than seeing bridges and ferries in opposition to one another, we have presented them in the case of Zhoushan Archipelago as complementary components of a complex transport network.

By comprehensively presenting the cross-sea transport links in Zhoushan Archipelago, we have been able to provide empirical data that can serve as the basis for creating a counter-discourse that avoids oppositional understandings of fixed links and waterborne transport. Future research should seek to assess how Zhoushan Archipelago as well as other islands and archipelagos come to host similarly complex (and potentially complementary) transport networks and how such networks affect the ways in which the geography of islandness is practiced.

\section{Funding}

The authors disclosed receipt of the following financial support for the research, authorship, and/or publication of this article: (1) General Program, Government Sponsored Research, National Nature Science Foundation of China, No.: 51878592; (2) Zhejiang Province Philosophy and Social Sciences Planning Countermeasures Application Subject, No.: 19NDYD32YB; (3) Basic Public Welfare Research Program of Zhejiang Province, No.: LGN20E080002.

\section{References}

Andersen, S. N., Nilsen, Ø. L., Gutiérrez, M. D., Tørset, T., 2016. Impacts on land use characteristics from fixed link projects: four case studies from Norway. Transportation Research Procedia 13: 145-154.

Baldacchino, G., 2020. How far can one go? How distance matters in island development. Island Studies Journal 15: 25-42.

Baldacchino, G., 2007. Fixed links and the engagement of islandness: reviewing the impact of the Confederation Bridge. The Canadian Geographer/Le Géographe Canadien. 51: 323-336.

Bingenheimer, M., 2016. Island of Guanyin: Mount Putuo and its gazetteers. Oxford University Press, Oxford.

Bråthen, S., 2001. Do fixed links affect local industry? A Norwegian case study. Journal of Transport Geography 9: 25-38. 
Castanho, R.A., Behradfar, A., Vulevic, A., Naranjo Gómez, J.M., 2020. Analyzing transportation sustainability in the Canary Islands archipelago. Infrastructures 5: 58 .

Chandler, D., Pugh, J., 2020. Islands of relationality and resilience: The shifting stakes of the Anthropocene. Area 52: 65-72.

Chen, Q., Dong, H., 2019. Sustainable development indicator systems for island cities: The case of Zhoushan Maritime Garden City. Island Studies Journal 14: 137-156.

China Daily, 2019. Railway connecting Ningbo, Zhoushan starts construction. China Daily, 5 March. http://zhoushan.chinadaily.com.cn/201903/05/c_342647.htm

Cooke, G. B., Petersen, B. K., 2019. A typology of the employment-education-location challenges facing rural island youth. Island Studies Journal 14: 101-124.

Gillis, J., 2004. Islands of the mind: how the human imagination created the Atlantic world. Palgrave Macmillan, Houndmills.

Gray, D., Farrington, J. H., Kagermeier, A., 2008. Geographies of rural transport. In Knowles, R., Shaw, J., Docherty, I. (Eds), Transport geographies: Mobilities, flows and spaces. Blackwell, Malden, pp. 102-119.

Gray, D., Shaw, J., Farrington, J., 2006. Community transport, social capital and social exclusion in rural areas. Area 38: 89-98.

Grydehøj, A., 2019. Critical approaches to island geography. Area 52: 2-5.

Grydehøj, A., 2017. A future of island studies. Island Studies Journal, 12(1): 3-16.

Grydehøj, A., Casagrande, M., 2019. Islands of connectivity: Archipelago relationality and transport infrastructure in Venice Lagoon. Area 52: 5664.

Grydehøj, A., Hayward, P., 2014. Social and economic effects of spatial distribution in island communities: Comparing the Isles of Scilly and Isle of Wight, UK. Journal of Marine and Island Cultures 3: 9-19.

Hayward, P., 2016. Towards an expanded concept of island studies. Shima 10: 1-7.

Hayward, P., 2015. The aquapelago and the estuarine city: reflections on Manhattan. Urban Island Studies 1: 81-95.

Hong, G., 2017. Locating Zhuhai between land and sea: A relational production of Zhuhai, China, as an island city. Island Studies Journal 12: 725.

Hong, G., 2020a. A critical-holistic approach to the place-specific geographies of inhabited river islands on the rural-urban fringe of inland China. Shima 14(2), 149-167.

Hong, G., 2020b. Islands of enclavization: Eco-cultural island tourism and the relational geographies of near-shore islands. Area 52: 47-55.

Knaap, T., Oosterhaven, J., 2003. Spatial economic impacts of transport infrastructure investments. In Pearman, A., Mackie, P., Nellthorp, J. (Eds), Transport projects, programmes and policies: evaluation needs and capabilities. Routledge, Ashgate, pp. 87-105.

Knowles, R., 2000. The Great Belt Fixed Link and Denmark's transition from inter-island sea to land transport. Geography: Journal of the Geographical Association 85: 345-354.

Krieg, L. J., 2018. Entangling (non)human isolation and connectivity: island nature conservation on lle aux Aigrettes, Mauritius. Island Studies Journal 13: 55-70.

Lama, P. D., 2018. Gendered consequences of mobility for adaptation in small island developing states: case studies from Maafushi and Kudafari in the Maldives. Island Studies Journal 13: 111-128.

Lee, S. H., Huang, W.H., Grydehøj, A., 2017. Relational geography of a border island: local development and compensatory destruction on Lieyu, Taiwan. Island Studies Journal 12: 97-112.

Leung, A., Tanko, M., Burke, M., Shui, C.S., 2017. Bridges, tunnels, and ferries: connectivity, transport, and the future of Hong Kong's outlying islands. Island Studies Journal 12: 61-82. 
Lu, H., Zhang, M., Sun, W., Li, W., 2018. Expansion analysis of Yangtze River Delta urban agglomeration using DMSP/OLS nighttime light imagery for 1993 to 2012. ISPRS International Journal of Geo-Information 7: 52.

Matas, A., Raymond, J. L., Roig, J. L., 2015. Wages and accessibility: the impact of transport infrastructure. Regional Studies 49: $1236-1254$.

Mendas, Z., 2015. Tracing socio-economic impact of ferry provision in Zadar island archipelago. Journal of Marine and Island Cultures 4: 10-26.

Moseley, M. J., 1979. Accessibility: the rural challenge. Methuen, London.

National Bureau of Statistics of China, 2011. Major figures on 2010 population census of China. China Statistical Press, Beijing.

Pan, Y., Zhai, M., Lin, L., Lin, Y., Cai, J., Deng, J. S., Wang, K., 2016. Characterizing the spatiotemporal evolutions and impact of rapid urbanization on island sustainable development. Habitat International 53: 215-227.

Pugh, J., 2018. Relationality and island studies in the Anthropocene. Island Studies Journal 13: 93-110.

Qiu, S., Yue, W., Zhang, H., Qi, J., 2017. Island ecosystem services value, land-use change, and the National New Area Policy in Zhoushan Archipelago, China. Island Studies Journal 12: 177-198.

Raadik Cottrell, J., Cottrell, S. P., 2019. Sense of place predictors of perceived effects of a proposed island to mainland bridge on future experience. Island Studies Journal 14: 187-204.

Ratter, B. M., 2018. Geography of small islands: outposts of globalisation. Cham: Springer.

Seedhouse, A., Johnson, R., Newbery, R., 2016. Potholes and pitfalls: the impact of rural transport on female entrepreneurs in Nigeria. Journal of Transport Geography 54: 140-147.

Sheng, N., Tang, U. W., Grydehøj, A., 2017. Urban morphology and urban fragmentation in Macau, China: island city development in the Pearl River Delta megacity region. Island Studies Journal 12: 199-212.

$\mathrm{Si}, \mathrm{K} ., 2019$ a. Ningbo-Zhoushan passes Shenzhen to become third largest container port globally. Seatrade Maritime News, 17 January. http://www.seatrade-maritime.com/news/asia/ningbo-zhoushan-passes-shenzhen-to-become-third-largest-container-port-globally.html

Si, K., 2019b. Shanghai retains crown of world's busiest container port. Seatrade Maritime News, 15 January. http://www.seatrademaritime.com/news/asia/shanghai-keeps-crown-of-world-s-busiest-container-port.html

Swaminathan, R., 2014. The epistemology of a sea view: mindscapes of space, power and value in Mumbai. Island Studies Journal 9: $277-292$.

Vannini, P., 2011. Constellations of ferry (im)mobility: islandness as the performance and politics of insulation and isolation. Cultural Geographies 18: 249-271.

Wu, Y., Zhang, T., Zhang, H., Pan, T., Ni, X., Grydehøj, A., Zhang, J., 2020a. Factors influencing the ecological security of island cities: A neighborhood-scale study of Zhoushan Island, China. Sustainable Cities and Society 55, 102029.

Wu, Y., Wang, S., Yang, J., Wu, S., You, H., Wang, Y., 2020b. Impact of land use on coastline change of island cities: A case of Zhoushan Island, China. Island Studies Journal 15(2). Epub ahead of print.

Yue, W., Qiu, S., Zhang, H., Qi, J., 2017. Migratory patterns and population redistribution in China's Zhoushan Archipelago in the context of rapid urbanization. Island Studies Journal 12: 45-60.

Zhang, H., Grydehøj, A., 2020. Locating the interstitial island: Integration of Zhoushan Archipelago into the Yangtze River Delta urban agglomeration. Urban Studies. Epub ahead of print.

Zhang, H., Xiao, Y., 2020. Planning island sustainable development policy based on the theory of ecosystem services: A case study of Zhoushan Archipelago, East China. Island Studies Journal 15: 237-252.

Zhang, M., Xiao, H., Sun, D., Li, Y., 2018. Spatial differences in and influences upon the sustainable development level of the Yangtze River Delta Urban Agglomeration in China. Sustainability 10: 411.

Zhoushan City Housing and Urban-Rural Development Bureau, 2015. 浙江舟山群岛新区（城市）总体规划（2012-2030年）.Zhoushan City Housing and Urban-Rural Development Bureau, 17 July. http://zszjj.zhoushan.gov.cn/art/2015/7/17/art_1604437_1776.html 